\title{
O papel da educação online na formação de adultos: o caso da Universidade Aberta
}

\author{
Glória Bastos, Rosa Maria Sequeira \\ \& Daniela Melaré Vieira Barros
}

Resumo:

Segundo documentos de organizações internacionais (UNESCO, OCDE, EU), a educação a distância e a aprendizagem online são modalidades que podem responder de forma mais cabal aos desafios que colocam as estratégias de cooperação europeia no âmbito da formação e aos objetivos estabelecidos para os estados-membros nesse contexto. No presente artigo analisam-se alguns desses documentos mais recentes e explana-se o papel da aprendizagem a distância (sobretudo ao nível do ensino superior) no quadro da educação de adultos e ao longo da vida, como um contributo central, em termos de inclusão social e de valorização pessoal e profissional dos cidadãos. Em face de quadros de referência europeus como o das competências em empreendedorismo e o das competências digitais, consideramos que o ensino online será mesmo a resposta mais adequada. Referimos ainda o papel singular que a Universidade Aberta de Portugal (UAb) tem no panorama do ensino superior português, enquanto instituição pública criada especificamente para atuar no contexto de educação a distância e analisamos algumas das formas pelas quais a UAb tem respondido aos desafios que the têm sido colocados, considerando a especificidade do seu perfil de estudante e a necessidade de criação de estratégias que conduzam ao cumprimento da sua importante função social.

Palavras-chave:

educação a distância online; educação de adultos; inclusão social e digital; Universidade Aberta. 


\section{The role of online education in adult education: the case of Universidade Aberta}

Abstract: According to documents from international organizations (UNESCO, OECD, EU), distance education and online learning can best respond to the challenges of European cooperation strategies for training and the objectives set for Member States in this area. This article examines some of these more recent documents and explores the role of distance learning (especially at tertiary level) in adult and lifelong learning as a central contribution in terms of inclusion and personal and professional enhancement of citizens. In the face of European reference frameworks such as entrepreneurship and digital skills, we believe that online learning is indeed the most appropriate answer. We also refer to the unique role that Universidade Aberta (UAb, Portuguese Open University) plays in the Portuguese higher education landscape, as a public institution created specifically to act in the context of distance education, and we analyze some of the ways in which UAb has responded to the challenges it has been facing, considering the specificity of the student profile and the need to create strategies properly to its important social function.

Keywords: online distance education; adult education; social and digital inclusion; Universidade Aberta.

\section{Le rôle de l'éducation en ligne dans l'éducation des adultes: le cas de I'Universidade Aberta}

Résumé: Selon des documents émanant d'organisations internationales (UNESCO, OCDE, UE), l'enseignement à distance et l'apprentissage en ligne peuvent le mieux répondre aux défis des stratégies de coopération européenne en matière de formation et aux objectifs fixés pour les États membres dans ce domaine. Cet article examine certains de ces documents plus récents et explore le rôle de l'apprentissage à distance (en particulier au niveau tertiaire) dans l'apprentissage des adultes et tout au long de la vie en tant que contribution essentielle en termes d'inclusion, valorisation sociale, personnelle et professionnelle des citoyens. Face aux cadres de référence européens tels que l'entrepreneuriat et les compétences digitales, nous pensons que l'apprentissage en ligne est en effet la réponse la plus adéquate. Nous évoquons également le rôle unique qui joue l' Universidade Aberta au Portugal (UAb) dans le panorama de l'enseignement supérieur portugais, en tant qu'institution publique créée spécifiquement pour agir dans le contexte de la formation à distance, et nous analysons certaines des réponses apportées par l'UAb aux défis auxquels elle est confrontée, en tenant compte de la spécificité de leur profil d'étudiant et de la nécessité de créer des stratégies qui conduisent à la réalisation de leur importante fonction sociale.

Mots clé: éducation à distance et en ligne; éducation des adultes; inclusion sociale et numérique; Universidade Aberta.

\section{El papel de la educación en línea en la educación de adultos: el caso de la Universidade Aberta}

Resumen: Según documentos de organizaciones internacionales (UNESCO, OCDE, UE), la educación a distancia y el aprendizaje en línea pueden responder mejor a los desafíos de las estrategias de cooperación europea en materia de formación y a los objetivos establecidos para los Estados miembros en este ámbito. Este artículo examina algunos de estos documentos más recientes y explora el papel del aprendizaje a distancia (especialmente a nivel terciario) en la formación de adultos y a lo largo de la vida como una contribución central en términos de inclusión, mejora social, personal y profesional de los ciudadanos. Frente a los marcos de referencia europeos como el emprendimiento y las habilidades digitales, creemos que el aprendizaje en línea es la respuesta más adecuada. También nos referimos al papel único que desempeña la Universidade Aberta de Portugal (UAb) en el panorama de la educación superior portuguesa, como una institución pública creada específicamente para actuar en el contexto de la educación a distancia, y analizar algunas de las formas en que UAb ha respondido a los desafíos que ha enfrentado, consideración de la especificidad de su perfil de estudiante y la necesidad de crear estrategias que conduzcan al cumplimiento de su importante función social.

Palabras-clave: educación a distancia en línea; educación de personas adultas; inclusión social y digital; Universidade Aberta. 


\section{Introdução}

Os principais sistemas modernos de educação a distância iniciaram o seu percurso para um acesso alargado ao ensino superior nas décadas seguintes ao final da II Grande Guerra. Como marcos importantes, no contexto europeu, temos a criação da Open University, no Reino Unido (1969) e logo a seguir da UNED, Universidad Nacional de Educación a Distancia, em Espanha (1972), e da FernUniversität, na Alemanha (1974).

A promoção da educação para todos, com particular enfoque nos adultos que não têm ou não tiveram possibilidade de aceder ao ensino superior nas instituições convencionais (presenciais), liga-se à problemática do capital humano e ao papel da educação nesse contexto, reconhecendo-se que a qualificação dos recursos humanos tem impacto na economia e favorece o crescimento económico, a par do desenvolvimento social e cultural. Pode também associar-se a perspetivas de cariz mais humanista, com base no entendimento de que o acesso à educação (formal e não formal, e em qualquer nível de ensino) é um direito humano importante e ao seu papel na valorização pessoal e como vontade autónoma do indivíduo (cf., por exemplo, Lucio-Villegas, 2018).

Alain Tait, profundo conhecedor da história da educação a distância, identifica uma das razões nucleares para o aparecimento das universidades abertas: "highly political institutions invented because of the inadequacy of the higher education sector to meet the challenge of modernity, defined both in terms of who is to be included in the goods of society and what society needs in terms of human capital” (2008, p. 92). O desenvolvimento e a justiça social estão, pois, no cerne da sua missão (p. 93). E acrescenta ainda uma ideia crucial: o papel e a intervenção que as universidades abertas têm vindo a assumir na mudança do sistema de ensino superior em termos de qualidade e inovação (p. 92).

Estas questões são elementos centrais quando pensamos no ensino superior a distância na atualidade e no seu papel social e inovador em termos organizativos e pedagógicos (nomeadamente, na forma como coloca o estudante adulto no centro do processo de aprendizagem e como dá particular atenção aos materiais de aprendizagem e aos processos avaliativos). As diversas iniciativas, orientações e recomendações que entretanto a nível internacional (com repercussões nacionais) têm sido concebidas no sentido da educação ao longo da vida e da imprescindibilidade de uma educação para todos sublinham em diferentes momentos a relevância da educação a distância enquanto modalidade de ensino que permite cumprir cabalmente esses desígnios.

Marcella Milano (2012) dá-nos conta da deslocação que tem ocorrido, em especial nos documentos de entidades internacionais, da noção "educação de adultos" para "aprendizagem ao longo da vida" e das suas implicações políticas e económicas. Neste texto não prescindimos das duas designações: (i) educação de adultos, porque 
o foco são populações maduras e isso molda os contextos de ensino e de aprendizagem online; (ii) educação ou aprendizagem ao longo da vida, porque cada vez mais o envolvimento dos indivíduos em processos e atividades de aprendizagem (formal, não formal ou informal) tem-se constituído como um sistema contínuo.

\section{Desafios na educação online na aprendizagem de adultos}

\section{Orientações para a educação de adultos e ao longo da vida}

A Declaração de Incheon apresenta um título sugestivo: Educação 2030: rumo a uma educação de qualidade inclusiva, equitativa e à educação ao longo da vida para todos (UNESCO, 2015). Para alcançar os objetivos propostos, os percursos de aprendizagem flexíveis e as tecnologias surgem com especial relevância: "Information and communication technologies (ICTs) must be harnessed to strengthen education systems, knowledge dissemination, information access, quality and effective learning, and more effective service provision" (p. 8). Neste cenário, a educação online, sendo um tema que vem sendo desenvolvido já de longa data, ganha mais visibilidade ao estar na agenda dos organismos internacionais e no contexto europeu de maneira muito contundente. A educação online permite, de facto, concretizar a flexibilidade e o alargamento do acesso ao ensino superior, conforme destacam documentos mais recentes da ONU, UNESCO e União Europeia.

Em 2006, o Parlamento Europeu e o Conselho da Europa produziram um conjunto de recomendações para a formação ao Longo da Vida. Estas recomendações deram origem a um quadro de referência europeu de competências básicas neste âmbito, que sofreu uma revisão em 2016 e em maio de 2018 foi substituído por um novo documento. Este quadro de referência não serve apenas de base de trabalho para uma educação orientada por competências, mas também intenta fornecer orientações às políticas nacionais no âmbito de um quadro estratégico para a cooperação europeia no que respeita à formação de adultos. As mudanças entretanto ocorridas na sociedade, no sentido de uma maior automatização dos trabalhos e de um maior papel das tecnologias, justificavam esta revisão que tinha de ser ajustada também com outros documentos entretanto produzidos quer pelo Conselho da Europa, quer pela OCDE, quer ainda por outros organismos como a UNESCO. Desde então outros documentos foram dando atenção a aspetos particulares da Formação ao Longo da Vida, nomeadamente O Pilar Europeu dos Direitos Sociais (2017), que consigna o direito à formação e requalificação para a empregabilidade, desenvolvimento pessoal, saúde e inclusão social; a recomendação 2016/C484/01 sobre as novas oportunidades; o Quadro Europeu de Referência para as Competências Digitais, com uma lista de 21 competências (https:// ec.europa.eu/jrc/en/digcomp/digital-competence-framework); ou o Quadro Europeu de Referência para as competências em empreendedorismo. 
No que respeita a Portugal, de acordo com o Monitor de Educação e Formação de 2018, o país continua a apresentar níveis baixos de participação em atividades de formação, situação que constitui um desafio a reter, na medida em que mais de metade da população adulta possui um nível baixo de habilitações: (in: https://ec.europa.eu/ portugal/news/education-training-europe-2018_pt).

Entre os objetivos comuns que a União Europeia estabelece para os seus estados membros para 2020 contam-se o aumento da média de participação de adultos na Formação ao Longo da Vida e na educação terciária (https://ec.europa.eu/eurostat/ web/education-and-training/eu-benchmarks. Ora se a percentagem de indivíduos que concluíram o ensino superior em Portugal aumentou para 39,9 \%, atingindo quase o objetivo de 40 \% acordado para 2020, já a participação de adultos na Formação ao Longo da Vida encontra-se abaixo da média estabelecida (10,3\% contra os 15\%).

Também a avaliação da OCDE ao sistema de ensino superior em Portugal aponta inconsistências entre as diferentes estratégias do país para desenvolver o ensino superior e a investigação. Um aspeto sublinhado reside exatamente na necessidade de alargar as possibilidades a adultos de acesso ao ensino superior. Portugal apresenta 9\% de estudantes com mais de 25 anos no ensino superior, percentagem abaixo dos 18\% da média europeia (http://www.oecd.org/portugal/oecd-review-of-higher-education-research-and-innovation-portugal-9789264308138-en.htm).

A flexibilidade é considerada, em muitos destes documentos, um fator chave para o desenvolvimento em educação. A aprendizagem flexível é comummente identificada como um meio para lidar com a evolução da composição da população estudantil, para assim acomodar as necessidades e constrangimentos de uma população mais diversificada. Essa busca prática da convergência da flexibilidade para a ação na educação é concretizada, nomeadamente, através da educação a distância.

Segundo a Comissão Europeia (2011), a flexibilidade da aprendizagem e dos métodos de ensino pode ser um meio para melhorar a qualidade e a pertinência dos estudos superiores, ao mesmo tempo que aumenta o número de estudantes, alarga a participação a diversos grupos e combate o abandono. Neste âmbito, os Estados membros da União Europeia foram convidados a incentivar uma maior variedade de formas de aprendizagem, como o tempo parcial; à distância; através de módulos; formação contínua para adultos; entre outras. Estas orientações, retomadas em diferentes momentos, foram recentemente apontadas como uma das principais prioridades: "melhorar a igualdade no acesso à educação inclusiva e de alta qualidade, assim como a formação em todas as fases da vida, desde a infância ao ensino superior e à educação de adultos" (2019, p. 29).

Outro investimento nesta área da educação e formação de jovens e adultos em cenários online tem um importante momento de incentivo em Portugal quando, em abril de 2017, o governo apresenta a Iniciativa Nacional Competências Digitais e.2030 
(INCoDe.2030), "para melhorar as competências em tecnologias de informação e comunicação, que define medidas para a inclusão, educação, qualificação profissional, especialização e investigação científica até 2030" (Governo de Portugal, 2017). Esta iniciativa, influenciada pela dinâmica europeia, apresenta-se como um programa integrado de competências digitais para Portugal, com foco em três desafios: garantir a literacia e a inclusão digitais para o exercício pleno da cidadania; estimular a especialização em tecnologias e aplicações digitais para a qualificação do emprego e uma economia de maior valor acrescentado; e produzir novos conhecimentos em cooperação internacional.

\section{O estudante adulto e a aprendizagem}

Os alunos adultos podem ser definidos como aqueles que continuam a sua educação equilibrando a família e o trabalho (Kahu et al., 2013), havendo variações na idade de referência (Richardson \& King, 1998). Por exemplo, no caso da Universidade Aberta a idade mínima de entrada é 21 anos, mas temos também a linha dos 23 anos para o acesso ao ensino superior do chamado público "não tradicional", sendo que os estudos da OCDE fixam nos 25 anos as análises referentes à escolaridade da população adulta.

Embora os estudos sobre os estudantes adultos se centrem mais nas suas dificuldades e necessidades específicas do que nas mais-valias que trazem (Richardson \& King, 1998), é um facto que o estudante maduro apresenta características que o distinguem do aluno tradicional: está ciente das razões para estudar e do que precisa aprender (Knowles, 1996), distinguindo-se de outros perfis de estudantes em termos das responsabilidades nas suas vidas diárias, das motivações e expetativas. Tudo isto influencia a sua experiência de aprendizagem (Cercone, 2008; Gravani, 2015), por isso há necessidade de um ambiente educacional onde eles possam determinar os seus próprios percursos de aprendizagem, partilhar as suas ideias e sustentar o seu processo educativo em simultâneo com a sua vida privada. Assim, o ambiente de educação a distância online oferece-Ihes as oportunidades e os meios adequados.

A educação a distância proporciona aos estudantes adultos a vantagem da aprendizagem ao longo da vida devido à sua flexibilidade, sendo entendida como "teaching and planned learning in which teaching generally occurs in a different place from learning, requiring communication through technologies as well as special institutional organization" (Moore \& Kearsley, 2011, p. 2). Esta definição, que tem sido retomada em muitas circunstâncias, coloca a tónica em diversos elementos que se constituem como questões centrais quando se fala em educação a distância, designadamente a separação física e temporal entre estudantes e professores e a comunicação mediada através 
da tecnologia. Mas acrescenta um outro ponto menos considerado: é necessário uma organização institucional especial, que possa responder de forma eficaz aos requisitos de uma verdadeira educação em rede.

Neste contexto, e no sentido de serem propostos percursos formativos adequados, convém atender ao conceito de andragogia, como conceção de uma abordagem de ensino para adultos que levou à perceção de novos aspetos sobre a aprendizagem e as capacidades desses estudantes. Evidenciando o papel da autoaprendizagem ou aprendizagem autodeterminada, é definida por Knowles (1970, p. 103) como "The process in which individuals take the initiative, with or without the help of others, in diagnosing their learning needs, formulating learning goals, identifying human and material resources for learning, choosing and implementing learning strategies, and evaluating learning outcomes".

Procurando descrever de forma mais clara a noção da aprendizagem autodeterminada, surge a heutagogia, proposta por Stewart Hase e Chris Kenyon (2007). A heutagogia caracteriza-se pelo foco na aprendizagem autodeterminada, em que o estudante determina os conteúdos da sua aprendizagem, valorizando-se neste processo a experiência prática, e tem vindo a ser explorada no campo da educação a distância (Blaschke, 2012), tendo em atenção as diferentes proximidades que se podem identificar.

A partir destas abordagens e em cenários online e assíncronos, destacamos que as diferentes formas de uso dos contextos virtuais de aprendizagem ajudam-nos a identificar a importância de estratégias didático-pedagógicas diversificadas, nomeadamente através da utilização de diferentes recursos e ferramentas digitais, exatamente pelas possibilidades que podem oferecer para atender às diferentes preferências e individualidades.

Embora a teoria clássica dos estilos de aprendizagem tenha sido concebida a partir de referenciais de presencialidade e não de cenários assíncronos, podemos identificar diferentes formas de apropriação dos contextos virtuais (cf. Barros, 2014; Barros, 2018). Assim, esta teoria não deixa de fornecer algumas diretrizes para compreendermos melhor como aprender e como ensinar, também em cenários online. Por exemplo: maior atenção às individualidades dos estudantes; ênfase nos processos metodológicos; desenvolvimento de estratégias didático-pedagógicas diversas com base nos estilos de aprendizagem; ampliação das possibilidades de avaliação do aluno; melhoria das possibilidades de aprendizagem no processo educativo a distância, através da oferta diversificadas de atividades e materiais didáticos com impacto positivo na aprendizagem (Barros et al., 2010; Ota, Araújo Júnior \& Barros, 2017). 


\section{Educação a distância online e inclusão}

Como foi já mencionado, um dos fatores que justifica o investimento na educação de adultos e ao longo da vida prende-se com o capital humano, a inclusão social e a educação para a cidadania, uma prioridade da União Europeia na atualidade. Como se procurou explanar, a aprendizagem a distância cria condições favoráveis para a concretização destes objetivos. Fortalecendo a visão de uma sociedade aprendente, a educação ao longo da vida em espaços de aprendizagem online favorece igualmente o domínio de ferramentas essenciais para uma cidadania digital e para uma maior confiança profissional em que os contextos digitais assumem cada vez maior protagonismo (Novo, Bastos e Vasconcelos, 2016). Outros estudos testemunham o impacto da educação de adultos em contextos online e blended para a inclusão social em articulação com o capital social (Cocquyt et al., 2012).

Em simultâneo, reconhece-se que as tecnologias de comunicação e informação, em especial as tecnologias móveis, trouxeram novos públicos para a educação superior, públicos não-tradicionais, mas colocaram também novos desafios à problemática da inclusão social (Traxler, 2016) na medida em que as próprias instituições devem definir a forma como se apropriam dos novos usos tecnológicos no sentido de poderem abranger de forma mais extensiva essas populações. Consideramos que as tecnologias, enquanto recursos indissociáveis do contexto de aprendizagem atual, devem estar em estreita articulação com um pensamento pedagógico claro especialmente na educação a distância online. Só desta forma podem estar ao serviço do desenvolvimento pessoal e social e contribuir para o sucesso dos processos de aprendizagem.

\section{A Universidade Aberta: um caso de inovação e inclusão no contexto do ensino a distância online}

Iremos considerar seguidamente o papel da aprendizagem a distância ao nível do ensino superior, no quadro da educação de adultos e ao longo da vida, como um contributo central, em termos de inclusão social e de valorização pessoal e profissional dos cidadãos, atendendo ao caso da Universidade Aberta (UAb) e analisando as formas como esta tem respondido aos desafios que the têm sido colocados.

A UAb tem um papel singular no panorama do ensino superior português, enquanto instituição pública criada especificamente para atuar no contexto de educação a distância. A especificidade do seu perfil de estudante e a necessidade de criação de respostas que conduzam ao cumprimento da sua importante função social têm resultado na construção de estratégias de ação que merecem, do nosso ponto de vista, uma leitura atenta, pela dimensão de inovação que comportam quando comparada com outras experiências similares. O novo quadro legislativo para a educação a distância, 
entretanto aprovado ${ }^{1}$, torna mais relevante esta análise, pelos contributos que também pode trazer para apoiar a definição de políticas futuras nesta área.

Criada em 1988, num contexto em que a qualificação da população adulta constituía uma urgência nacional, a história da Universidade Aberta está intimamente ligada a uma intervenção ampla na capacitação de adultos ativos, a nível da sua formação superior. Os esforços para alcançar públicos mais alargados foram intensificados a partir do início do novo milénio, através das primeiras experiências com contextos de aprendizagem online.

O reconhecimento de que o trabalho com populações adultas requer uma abordagem específica e a necessidade de se identificarem e aplicarem metodologias que facilitassem a aprendizagem a distância online conduziram a UAb à conceção de um modelo pedagógico próprio. O Modelo Pedagógico Virtual (MPV®) da Universidade Aberta está fundamentado em quatro grandes linhas: aprendizagem centrada no estudante, primado da flexibilidade, da interação e da inclusão digital. Estas linhas norteiam a organização do ensino, o papel do estudante e do professor, a planificação, conceção e gestão das atividades de aprendizagem a propor aos estudantes, a tipologia de materiais a desenvolver e a natureza da avaliação das competências adquiridas (Pereira et al., 2007; Quintas Mendes et al., 2018).

No panorama mais alargado dos países falantes de português, a educação a distância online e em rede constitui a única forma de facultar às populações adultas o acesso à educação e à formação de qualidade, segundo os padrões do trabalho pedagógico já expostos, que partem da centralidade do estudante, pessoa adulta quase sempre já inserida no mercado de trabalho. Por outro lado, o tipo de aprendizagem que adquire particular relevância na sociedade em rede é a que permite ao estudante trabalhar de forma flexível e em autonomia. Em ambientes de aprendizagem online temos também um estudante que ganha confiança crescente face aos ambientes digitais, revelando maior competência nessas áreas fundamentais na sociedade atual (Bastos, 2018). De facto, nos ambientes de aprendizagem digitais vivem-se os processos de pesquisa em contexto real e significativo, articulando-se muitas vezes com os próprios contextos profissionais.

A construção de percursos formativos com flexibilidade nos espaços e tempos de aprendizagem e o uso inteligente dos múltiplos recursos disponíveis em suportes diversos são razões pelas quais a UAb é cada vez mais a primeira escolha de muitos dos seus estudantes. E perante públicos específicos, há a necessidade de planear ambientes e processos educacionais adequados. Um exemplo de um planeamento para um público específico é o CQES - Curso de Qualificação para o Ensino Superior, que iremos expor seguidamente. 


\section{Públicos específicos e respostas adaptadas: o "Curso de Qualificação para os Estudos Superiores"}

Em 2010, no âmbito de um protocolo de cooperação estabelecido entre a UAb e a Agência Nacional para a Qualificação (ANQ), é concebido um curso que intenta promover a igualdade de oportunidades, o "Curso de Qualificação para os Estudos Superiores" (CQES). O protocolo visava responder às indicações de Bolonha na promoção da equidade e na desejada abertura da universidade a novos públicos, reconhecendo e creditando competências adquiridas em contexto pessoal e profissional. As eventuais lacunas de formação, resultantes da ausência de um percurso escolar regular por estes novos públicos que se pretendiam captar, poderiam comprometer quer o seu acesso ao ensino superior, quer a frequência bem-sucedida de cursos universitários a adultos motivados para prosseguir a sua formação.

Foi a este problema que o CQES pretendia responder. Assim, foi concebido para dar formação de base em áreas do conhecimento consideradas fundamentais, destinando-se a todos aqueles que pretendiam preparar-se de modo consistente para ingressar numa instituição de ensino superior e frequentá-la com sucesso.

Em relação à sua composição curricular, o curso foi gizado a partir de uma oferta pedagógica de banda relativamente larga, complementada por uma escolha de tipo vocacional, no total de três unidades curriculares, sendo obrigatória uma em língua materna e havendo duas opcionais nas áreas científicas do curso que o estudante pretendia frequentar. Esta composição curricular pretendia habilitar os estudantes com competências que lhes proporcionassem solidez de atuação, nomeadamente no domínio linguístico, de modo a que pudessem interagir adequadamente, e no domínio cognitivo e organizacional ligado às técnicas de aprendizagem e utilização adequada de recursos para uma aprendizagem autónoma, de modo a dominar adequadamente fontes de informação e instrumentos de trabalho próprios das disciplinas que compunham o curso.

Esta estrutura curricular tem-se mantido ao longo do tempo, tendo havido, porém, alguns ajustes. No seu primeiro momento, este curso foi concebido como um curso curto não formal, com a duração de nove semanas em que o sucesso na frequência do curso era comprovado pelo sucesso no exame de admissão à universidade. Após alguns anos de funcionamento, considerou-se necessário que o curso tivesse uma maior duração, passando, por conseguinte, de nove a treze semanas (um semestre) e tornou-se formal com trabalhos de avaliação contínua e exame final de acordo com o Modelo Pedagógico da Universidade Aberta. Entre 2011-2012 e 2017-2018 frequentaram este curso (na sua totalidade ou em unidades curriculares isoladas) mais de dois mil estudantes, que beneficiaram, por esta via, de uma possibilidade de consolidação de conhecimentos e de competências com vista ao prosseguimento de estudos de nível superior. 
Como nota complementar, refira-se que atualmente, no caso dos estudantes que se vierem a inscrever numa licenciatura da Universidade Aberta, os créditos obtidos no Curso (12 ECTS) poderão ser creditados a pedido do estudante, em qualquer altura do seu percurso académico, na respetiva licenciatura em substituição de unidades curriculares de caráter opcional a definir pela coordenação dos cursos, tendo em atenção que a creditação de competências numa determinada área científica pressupõe que o nível a que serão lecionadas as unidades curriculares do CQES terá de ser análogo ao das disciplinas do primeiro ano de uma licenciatura, em particular na demonstração da capacidade de mobilizar adequadamente diversos conhecimentos prévios e de selecionar e integrar esses conhecimentos perante uma determinada questão ou problema, próprios dos conteúdos curriculares das unidades curriculares envolvidas.

\section{Reflexões Finais}

No quadro de um mundo em mudança, nomeadamente no plano social e económico, mas também demográfico, sobretudo nas sociedades ocidentais, com um envelhecimento acentuado da população, o envolvimento dos adultos em atividades de formação, com objetivos pessoais diversos, constitui um desafio permanente. Como se procurou salientar, cada vez mais a resposta está na oferta de percursos flexíveis e personalizados e a educação a distância online emerge, na verdade, como o meio mais adequado para responder a esta situação. Em paralelo, surge ainda a permanente necessidade de qualificação de largas franjas de população adulta com dificuldades de acesso à educação, nomeadamente nos países em desenvolvimento.

No contexto europeu, o reforço das competências digitais tem surgido como um imperativo, com ênfase para a sua ligação ao empreendedorismo e à cidadania, com a conceção de quadros de referência destinados a cidadãos comuns, organizações educativas e consumidores. Uma vez mais, a aprendizagem online permite, a partir de contextos reais de uso das tecnologias digitais, o desenvolvimento dessas competências.

O caso da Universidade Aberta, nomeadamente através da conceção de um Modelo Pedagógico próprio (Pereira et al., 2007; Quintas Mendes et al., 2018) e da criação de ofertas formativas visando o reforço da inclusão social, pretendendo assim responder com qualidade às especificidades assinaladas do estudante adulto, constitui um exemplo de boas práticas que foi já reconhecido internacionalmente. A aprendizagem colaborativa e autorregulada, a diferenciação e personalização são, desde há muito, características dos seus contextos virtuais de aprendizagem. E convém destacar, sobretudo face às características do mundo atual, fortemente digital, que este tipo de aprendizagem fornece o apoio e a orientação que possibilita aos adultos desenvolverem as suas competências digitais que poderão noutras ocasiões usar em contextos diversos, por exemplo de intervenção cidadã. 


\section{Notas}

${ }^{1}$ Decreto-Lei aprovado em reunião de Conselho de Ministros de 2 de agosto de 2019. No momento da escrita deste artigo o texto final ainda não foi publicado em Diário da República.

\section{Referências Bibliográficas}

Barros, D. M. V. (2014). Estilos de Aprendizagem e o Uso das Tecnologias. Santo Tirso: De Facto Editores.

Barros, D. M. V., Bianchi, A. M. Z., Nunes, J. S., Cavellucci, L. \& Valadas, S. (2010). Estilos de Aprendizagem e Educação a Distância: algumas perguntas e respostas. Journal of Learning Styles, 5 (5), 1-10.

Bastos, G. (2018). Universidade Aberta: percursos de afirmação e inovação pedagógica. In Carvalho, Madalena (org.). Acesso Aberto: da visão à ação: contextos, cenários e práticas. Lisboa: Universidade Aberta.

Blaschke, L. M. (2012). Heutagogy and Lifelong Learning: A Review of Heutagogical Practice and SelfDetermined Learning, IRRODL, The International Review of research in Open and Distance Learning, 13(1), 56-71.

Cercone, K. (2008). Characteristics of Adult Learners with Implications for Online Learning Design. AACE Journal, 16(2), 137-159. Acedido em https://www.learntechlib.org/primary/p/24286.

Cocquyt, C., Diep, N. A., Zhu, C., De Greef, M. \& Vanwing, T. (2017). Examining social inclusion and social capital among adult learners in blended and online learning environments. European Journal for Research on the Education and Learning of Adults, 8(1), 77-101.

COM (2011). Communication from the Commission to the European Parliament, the Council, the European Economic and Social Committee and the Committee of the Regions on 'Supporting growth and jobs - an agenda for the modernization of Europe's higher education systems. Acedido em: http://eur-lex.europa.eu/legal-content/EN/TXT/?uri=LEGISSUM:ef0030.

COM (2019). Reflection Paper Towards a Sustainable Europe by 2030. COM/2019/22 final. Acedido em https://eur-lex.europa.eu/legal-content/PT/TXT/PDF/?uri=CELEX:52019DC0022\&qid=1567001 579484\&from $=\mathrm{EN}$.

Governo de Portugal (2017). Portugal INCoDe.2030: Iniciativa Nacional Competências Digitais e.2030. Acedido em http://www.incode2030.gov.pt/sites/default/files/uploads/attachments/incode2030_ final_28mar17.pdf.

Gravani, M. N. (2015). Adult learning in a distance education context: theoretical and methodological challenges. International Journal of Lifelong Education, 34(2), 172-193. Doi: 10.1080/02601370.2014.982728.

Hase. S \& C. Kenyon (2007). Heutagogy: A Child of Complexity Theory in Complicity. An International Journal of Complexity and Education, 4(1), 111-118. Acedido em https://www.researchgate.net/ publication/37357676_Heutagogy_A_child_of_complexity_theory. 
Incheon Declaration. Education 2030: Towards inclusive and equitable quality education and lifelong learning for all. Republic of Korea For The World Education Forum (2015). Acedido em http:// unesdoc.unesco.org/images/0023/002338/233813m.pdf.

Knowles, M. (1970). The modern Practice of Adult Education: Andragogy versus Pedagogy. New York: Associated Press.

Knowles, M. (1996). Adult Learning. In Robert L. Craig (Ed.), The ASTD Training and Development Handbook (pp. 253-264). NY: McGraw-Hill.

Kahu, E. R, Stephens, C., Zepke, N. \& Leach, L. (2014). Space and time to engage: mature-aged distance students learn to fit study into their lives. Int. J. of Lifelong Education, 33(4), 523-540 doi: http://dx.doi.org/10.1080/02601370.2014.884177.

Lucio-Villegas, E. (2018). ¿Existe siquiera la educación de personas adultas? Reflexiones sobre un concepto diverso, Revista Lusófona de Educação, 42, 177-191. doi: 10.24140/issn.1645-7250. rle42.12.

Milana, M. (2012). Political globalization and the shift from adult education to lifelong learning. European journal for Research on the Education and Learning of Adults. 3(2), 103-117.

Novo, A., Bastos, G. \& Vasconcelos, A. I. (2016). Effects of a virtual learning environment on librarian's information literacy and digital literacy competences. S. Kurbanoğlu, J. Boustany, S. Špiranec, E. Grassian, D. Mizrachi \& L. Roy (Eds.). Information Literacy: Key to an Inclusive Society, Springer. Doi: 10.1007/978-3-319-52162-6.

Ota, M. A., Araújo Júnior, C. F. \& Barros, D. M. V. (2017). Estilos de aprendizagem em ambientes virtuais: cenários de investigação na educação superior, Educação, Formação e Tecnologias, Universidade do Minho, 10(1) p. 47-58. Acedido em http://eft.educom.pt/index.php/eft .

Pereira, A., Quintas Mendes, A., Morgado, L., Amante, L.\& Bidarra, J. (2007). Modelo Pedagógico Virtual da Universidade Aberta, Lisboa, Universidade Aberta.

Quintas Mendes, A., Bastos, G., Amante, L., Aires, L. L. \& Cardoso, T. (2018). Modelo Pedagógico Virtual. Cenários de Desenvolvimento. Lisboa: Universidade Aberta.

Tait, A. (2008). What are open universities for?, Open Learning: The Journal of Open, Distance and e-Learning, 23, (2), 85-93.

Traxler, J. (2016). Inclusion in an age of mobility. Research in Learning Technology, Vol. 24. Doi: http:// dx.doi.org/10.3402/rlt.v24.31372.

\section{Glória Bastos}

Universidade Aberta, Departamento de Educação e Ensino a Distância CEMRI - Centro de Investigação em Migrações e Relações Interculturais e LE@D - Laboratório de Educação a Distância e e-Learning ORCID: https://orcid.org/0000-0002-1432-225X 
Rosa Maria Sequeira

Universidade Aberta, Departamento de Humanidades Grupo de investigação em Comunicação Intercultural do CEMRI

Email: Rosa.Sequeira@uab.pt

ORCID: https://orcid.org/0000-0001-7501-8780

Daniela Melaré Vieira Barros

Universidade Aberta, Departamento de Educação e Ensino a Distância CEISXX e LE@D - Laboratório de Educação a Distância e e-Learning

Email: dbarros@uab.pt

ORCID: https://orcid.org/0000-0002-1412-2231

\section{Correspondência}

Glória Bastos

Universidade Aberta, Departamento de Educação e Ensino a Distância

Rua da Escola Politécnica, 147, 1269-001- Lisboa

Data de submissão: Setembro 2018

Data de avaliação: Dezembro 2018

Data de publicação: Setembro 2019 